\title{
Screening and identification of differentially expressed serum proteins in patients with vitiligo using two-dimensional gel electrophoresis coupled with mass spectrometry
}

\author{
YI-LEI LI, RUI-QUN QI, YANG YANG, HE-XIAO WANG, HANG-HANG JIANG, ZHENG-XIU LI, \\ BI-HUAN XIAO, SONG ZHENG, YU-XIAO HONG, JIU-HONG LI, HONG-DUO CHEN and XING-HUA GAO \\ Department of Dermatology, The First Hospital of China Medical University, Shenyang, Liaoning 110001, P.R. China
}

Received April 9, 2017; Accepted November 22, 2017

DOI: $10.3892 / \mathrm{mmr} .2017 .8159$

\begin{abstract}
In the clinic, vitiligo is characterized by two stages: Stable and progressive. The pathogenesis of vitiligo is still not clear. Here, we identified serum markers of vitiligo by screening for differentially expressed proteins in patients with vitiligo compared to healthy individuals. Serum samples were collected from patients with vitiligo $(n=10$ for both the stable and progressive stages) and healthy individuals $(n=10)$. Two-dimensional gel electrophoresis followed by matrix-assisted laser desorption/ionization time-of-flight mass spectrometry and western blotting were used to validate the differential expression of the proteins in the serum $(n=20$ each, at both stages for patients and healthy individuals). A total of 48 differentially expressed proteins were identified by gel image analysis. There were 28 differentially expressed proteins in patients with progressive vitiligo (PV) and 13 differentially expressed proteins in patients with stable vitiligo (SV) compared with that in healthy individuals. Additionally, 7 differentially expressed proteins were identified in patients with PV compared with those in patients with SV. The western blotting results showed that Peroxiredoxin-6, apolipoprotein L1, apolipoprotein E and mannose-binding protein were differentially expressed in patients with different stages of vitiligo. Our results showed that change serum levels of several proteins might be useful as biomarkers or in understanding the pathogenesis of vitiligo.
\end{abstract}

\section{Introduction}

Vitiligo is an acquired autoimmune disease characterized by primary, localized, or generalized white spots on the skin and

Correspondence to: Dr Xing-Hua Gao, Department of Dermatology, The First Hospital of China Medical University, 155 Nanjing Road, Shenyang, Liaoning 110001, P.R. China

E-mail: gaobarry@hotmail.com

Key words: serum proteomics, 2-DE, progressive-stage vitiligo, stable-stage vitiligo mucosae due to the destruction of epidermal melanocytes. Approximately $0.5-2 \%$ of the global population is affected by vitiligo (1), and the incidence of this disease in China is $0.1-2.7 \%$ (2). In the progressive stage of vitiligo, the emergence of new lesions or the enlargement of original lesions occurs from 6 weeks to 1 year, and the stable stage of the disease is reached only when lesions have been stable for more than 1 year or with spontaneous pigment regeneration. Vitiligo has a major impact on patient's quality of life. Autoimmunity, neural dysregulation, melanin self-destruction, or oxidative stress has been speculated to be involved in the pathogenesis of the disease (3). However, the true causes of damage to melanocytes are unclear.

Diagnosis of vitiligo primarily depends on clinical manifestation, and rarely on histopathological changes. So far, there have been no definite biomarkers for diagnosis, evaluation of disease progression, treatment response or prediction of prognosis. Infiltration by $\mathrm{CD}^{+} \mathrm{T}$ cells in the margin of lesions is suggestive of active vitiligo (4). Elevation of serum levels of chemokine ligands CXCL9 and CXCL10 were noted in both progressive and stable vitiligo (SV), especially in the former (5). IFN- $\gamma$ and TGF- $\beta$ were demonstrated to promote T-cell recruitment to the epidermis where melanocytes destruction may occur (6). Serum levels of IL-17 and oxidative stress products were claimed to have a possible association with progressive disease (7). However, the above findings require validation in further studies.

The two-dimensional gel electrophoresis (2-DE) followed by proteomic analysis is a useful tool for large-scale screening of disease-related differentially expressed proteins. By employing the technique, differentially expressed proteins in skin malignancy such as melanoma (8) and autoimmune disease such as systemic lupus erythematosus (SLE) were identified, which helped to clarify the pathophysiology and mechanism of the diseases (9). In this study, we used 2-DE followed by mass spectrometry (MS) to identify differentially expressed proteins in serum of both stages of vitiligo and the controls. Some representative differentially expressed proteins were further validated by western blotting. The present study provides a plausible basis for identifying the serum biomarker and studying the pathogenesis of vitiligo. 


\section{Materials and methods}

General treatment. Sixty patients were recruited from the Department of Dermatology, The First Hospital of China Medical University (Shenyang, China) from July 2015 to March 2016. The clinical diagnoses and classifications of all patients were consistent with the clinical classification of the curative effects of vitiligo formulated by the National Association of Integrative Medicine, Department of Dermatology, Committee of Pigment Disease (10). Among the samples from 30 patients representing each stage of vitiligo, 10 representatives of each stage were analyzed by 2-DE-MS, and 20 representatives were analyzed by western blotting. The average age in the progressive stage was $35.33 \pm 17.24$ years, and the average age in the stable stage was $34.32 \pm 16.28$ years. The control group consisted of 30 healthy volunteers with an average age of $35.43 \pm 15.26$ years. There were 13 man and 17 women in progressive vitiligo (PV), 12 man and 18 women in SV and 15 man and 15 women in the controls.

None of the patients were treated, and the controls had no other medical conditions, as confirmed by a comprehensive physical examination. This study was approved by the hospital ethics committee (no. 201202013-02), and all patients and healthy volunteers provided written informed consent for participation in the study.

Reagents. Urea was purchased from Gibco; Thermo Fisher Scientific, Inc. (Waltham, MA, USA). Propanesulfonic acid (CHAPS), ethylenediaminetetraacetic acid (EDTA), and dithiothreitol (DTT) were purchased from Promega Corporation (Madison, WI, USA). Iodoacetamide (IAM) was purchased from Promega Corporation, and sodium dodecyl sulfate (SDS), bromophenol blue, and a serum high-abundance protein depletion kit (ProteoPrep Blue Albumin and IgG Depletion kit) were purchased from Sigma-Aldrich; Merck KGaA (Darmstadt, Germany). Ammonium persulfate was purchased from Amresco, LLC (Solon, OH, USA), and acetonitrile (MS grade) was purchased from Thermo Fisher Scientific, Inc.

Collection and preparation of serum. A total of $5 \mathrm{ml}$ of blood was collected from the elbow vein of patients during the early morning under conditions of limosis. The samples were incubated at $4^{\circ} \mathrm{C}$ for $2 \mathrm{~h}$, centrifuged at $4^{\circ} \mathrm{C}$ and $3,000 \mathrm{x} \mathrm{g}$ for $10 \mathrm{~min}$ to separate the serum after self-coagulation, and then stored at $-80^{\circ} \mathrm{C}$. Depletion of high-abundance proteins was carried out using a serum high-abundance protein depletion kit according to the manufacturer's instructions.

2-DE and silver staining. For the first dimension isoelectric focusing gel, $120 \mu \mathrm{g}$ of total protein was loaded onto IPG prefabricated strips (24 cm, $\mathrm{pH} 4-7$; Bio-Rad Laboratories, Hercules, CA, USA). An isoelectric focusing instrument (GE Ettan IPGPhor3; GE Healthcare, Chicago, IL, USA) was used with the following protocol: $300 \mathrm{~V}$ for $30 \mathrm{~min}$, $700 \mathrm{~V}$ for $30 \mathrm{~min}, 1,500 \mathrm{~V}$ for $1.5 \mathrm{~h}, 9,000 \mathrm{~V}$ for $3 \mathrm{~h}$, and $9,000 \mathrm{~V}$ for $4 \mathrm{~h}$. This procedure was repeated three times for each individual sample. For the second dimension, the isoelectric focusing strip was used for SDS-polyacrylamide gel electrophoresis (PAGE) as follows. The IPG strips were placed in equilibration buffers I and II, shaken slowly for $15 \mathrm{~min}$, and then transferred to $30 \%$ gels and subjected to SDS-PAGE with the following protocol: $2 \mathrm{~W} / \mathrm{gel}$ for $45 \mathrm{~min}$ and $17 \mathrm{~W} /$ gel until the bromophenol blue ran to the end of the gel (approximately $4.5 \mathrm{~h}$ ). Silver staining was carried out according to standard procedures. Samples were stored at $4^{\circ} \mathrm{C}$ in $1 \%$ glacial acetic acid.

Gel image acquisition and analysis. The gel was scanned using a UMAX Powerlook 1100 scanner and analyzed with ImageMaster 2D platinum 5.0 (GE Healthcare). Background subtraction, homogenization, and matching were carried out for three gels from the same sample. Protein expression was compared between groups using t-tests. Differences with P-values of less than 0.05 were considered significant, and protein spots with an expression difference of at least 2-fold were defined as differentially expressed.

$M S$. Target protein spots were excised and subjected to in-gel enzymatic digestion. The peptides extracted from the protein spots were analyzed using an UltrafleX III TOF/TOF mass spectrometer (Bruker Corporation, Ettlingen, Germany) with the following settings: Ultraviolet (UV) wavelength, $355 \mathrm{~nm}$; repetition rate, $200 \mathrm{~Hz}$; acceleration voltage, 20,000 V; and optimal quality resolution, $1500 \mathrm{Da}$. Signals were collected from a scanning quality range of 700-3,200 $\mathrm{kDa}$. The peak of trypsin self-cleavage was used as an internal standard calibration in the MS experiments. The spectra of all experimental samples were obtained using the default mode. The Cytoscape plugin GlueGO and ReactomeFIViz were used to record the biological process GO term for the GO term enrichment analysis of differentially expressed proteins. P-values of $<0.05$ and FDR $<0.01$ were accepted as threshold values for GO enrichment. Identified Proteins were described in the tables by Accession, Score, PI, Mw, and \% coverage. Accession represent protein accession number in Uniprot database, Score represent MS score, PI represent Isoelectric point, Mw represent molecular weight, \% Coverage represent the percentage sequence coverage of the protein by the matched peptides.

Verification of protein expression by western blotting. The proteins of the serum samples were extracted by RIPA buffer (150 mM sodium chloride, $1.0 \% \mathrm{NP}-40,0.5 \%$ sodium deoxycholate $0.1 \%$ SDS, $50 \mathrm{mM}$ Tris, $\mathrm{pH}$ 8.0) and protein concentration was estimated by the Bradford protein assay (Bio-Rad Laboratories). Then the extracted proteins was boiled at $100^{\circ} \mathrm{C}$ for $5 \mathrm{~min}$. The serum samples of $20 \mathrm{PV}$ patients, $20 \mathrm{SV}$ patients and 20 healthy individuals were analyzed by WB experiment. Fifty micrograms of protein was separated by SDS-PAGE on $12 \%$ gels. The proteins were then transferred to polyvinylidene difluoride membranes at $4^{\circ} \mathrm{C}$ with a constant voltage of $120 \mathrm{~V}$. The membranes were then incubated with $5 \%$ skim milk powder at room temperature for $1 \mathrm{~h}$, followed by incubation with 5 primary antibodies (1:500-1:1,000) (Abcam, Cambridge, MA, USA): Anti-PRDX6 (cat. no. ab133348, 1:1,000 dilution; Abcam), anti-APOL1 (cat. no. ab108315, 1:1,000 dilution; Abcam), anti-APOE (cat. no. ab52607, 1:1,000 dilution; Abcam), anti-MBL2 (cat. no. ab189856, 1:500 dilution; Abcam) 
Table I. Identification of differentially expressed proteins of PV samples vs. control samples.

\begin{tabular}{|c|c|c|c|c|c|c|}
\hline Protein name & Accession & Score & PI & $\mathrm{Mw}, \mathrm{kDa}$ & Percent coverage, $\%$ & PV/C Ratio \\
\hline Ceruloplasmin & P00450 & 231 & 5.44 & 122,983 & 18 & -1000000 \\
\hline Vitronectin & P04004 & 201 & 5.55 & 55,069 & 25 & -1000000 \\
\hline Inter-alpha-trypsin inhibitor heavy & Q14624 & 233 & 6.51 & 103,521 & 21 & -2.33923 \\
\hline Kininogen-1 & P01042 & 360 & 6.34 & 72,996 & 33 & -1000000 \\
\hline Antithrombin-III & P01008 & 307 & 6.32 & 53,025 & 40 & -3.30293 \\
\hline Complement C4-B & P0C0L5 & 142 & 6.73 & 194,212 & 9 & -1000000 \\
\hline Ig mu chain $\mathrm{C}$ region & P01871 & 193 & 6.35 & 49,960 & 24 & -2.03550 \\
\hline Complement C3 & P01024 & 134 & 6.02 & 188,569 & 11 & -1000000 \\
\hline Apolipoprotein L1 & O14791 & 148 & 5.60 & 44,004 & 24 & -1000000 \\
\hline Inter-alpha-trypsin inhibitor heavy chain H4 & Q14624 & 199 & 6.51 & 103,521 & 11 & -11.94160 \\
\hline Apolipoprotein A-IV & P06727 & 110 & 5.28 & 45,371 & 33 & -1000000 \\
\hline Apolipoprotein A-I & P02647 & 361 & 5.56 & 30,759 & 68 & -1000000 \\
\hline Complement C4-A & P0C0L4 & 125 & 6.65 & 194,247 & 2 & -1000000 \\
\hline Keratin, type IIcytoskeletal 1 & P04264 & 198 & 6.11 & 76,359 & 14 & -1000000 \\
\hline Peroxiredoxin-6 & P30041 & 55 & 6.54 & 25,133 & 25 & -1000000 \\
\hline Ceruloplasmin & P00450 & 413 & 5.44 & 122,983 & 35 & 1.59452 \\
\hline Inter-alpha-trypsin inhibitor heavy chain $\mathrm{H} 4$ & Q14624 & 278 & 6.51 & 103,521 & 23 & 2.24357 \\
\hline Complement C4-A & P0C0L4 & 185 & 6.65 & 194,247 & 12 & 1.57939 \\
\hline Alpha-1B-glycoprotein & P04217 & 256 & 5.58 & 54,809 & 34 & 1.59039 \\
\hline Complement C3 & P01024 & 290 & 6.02 & 188,569 & 20 & 3.31003 \\
\hline Kininogen-1 & P01042 & 300 & 6.34 & 72,996 & 21 & 1000000 \\
\hline Ig alpha- 2 chain $C$ region & P01877 & 132 & 5.71 & 37,301 & 18 & 1.50594 \\
\hline Antithrombin-III & P01008 & 266 & 6.32 & 53,025 & 30 & 1.64382 \\
\hline Beta-2-glycoprotein 1 & P02749 & 107 & 8.34 & 39,584 & 15 & 1.52746 \\
\hline Ig gamma-4 chain $\mathrm{C}$ region & P01861 & 75 & 7.18 & 36,431 & 8 & 1.62011 \\
\hline Serotransferrin & P02787 & 164 & 6.81 & 79,280 & 21 & 2.02112 \\
\hline Complement factor I & P05156 & 187 & 7.72 & 68,071 & 19 & 2.20440 \\
\hline Apolipoprotein L1 & O14791 & 149 & 5.6 & 44,004 & 20 & 2.01092 \\
\hline
\end{tabular}

Mw, molecular weight; PI, Isoelectric point; PV, progressive vitiligo; C, control. The negative sign of PV/C ratio means downregulated protein expression. The positive sign of $\mathrm{PV} / \mathrm{C}$ ratio means upregulated protein expression. A ratio of 1000000 means that the protein only appeared in the progressive stage of vitiligo, but not in the healthy volunteers. A ratio of -1000000 means that the protein only appeared in the healthy volunteers, but not in the progressive stage of vitiligo.

and $\beta$-tubulin (cat. no. ab6046, 1:500 dilution; Abcam). The membranes were incubated with the primary antibodys at $4^{\circ} \mathrm{C}$ overnight for $10 \mathrm{~h}$. Next, the membranes were incubated with goat anti-rabbit IgG (HRP; cat. no. ab6721, 1:2,000 dilution; Abcam) followed by incubation with corresponding secondary antibodies at room temperature for $2 \mathrm{~h}$, Specific protein bands were visualized with the SuperSignal chemiluminescence system (Promega Corporation,) and imaged by X film.

Statistical analysis. All data were analyzed using Statistical Package for Science Software (SPSS) version 16.0 (SPSS, Inc., Chicago, IL, USA). The significant of difference were analysed by one-way analysis of variance (ANOVA). The gray value ratio of each band compared between groups (stable stage or progressive stage compared to control) was used to calculate the significance of differences by least significant difference (LSD) as a post hoc test. $\mathrm{P}<0.05$ was considered to indicate a statistically significant difference.

\section{Results}

2-DE-MS. A total of 48 proteins were obtained by MS analysis. Out of those 48, 26 were upregulated, and 22 were downregulated. There were 28 differentially expressed protein spots in the samples from patients with progressive stage vitiligo (13 upregulated and 15 downregulated) and 13 differentially expressed protein spots in the samples from patients with stable stage vitiligo (8 upregulated and 5 downregulated), compared to those in healthy individuals. The results of the 2-DE analysis comparing samples from patients with both stages of vitiligo to healthy individuals are shown in Tables I and II. The results of the 2-DE analysis comparing samples from patients with progressive stage vitiligo and patients with stable stage vitiligo are shown in Table III. A total of seven differentially expressed proteins were identified by MS analysis (5 upregulated, and 2 downregulated). 
Table II. Identification of differentially expressed proteins of SV samples vs. control samples.

\begin{tabular}{|c|c|c|c|c|c|c|}
\hline Protein name & Accession & Score & PI & $\mathrm{Mw}, \mathrm{kDa}$ & Percent Coverage, $\%$ & $\mathrm{SV} / \mathrm{C}$ ratio \\
\hline Kininogen-1 & P01042 & 191 & 6.34 & 72,996 & 11 & -1000000 \\
\hline Inter-alpha-trypsin inhibitor heavy chain $\mathrm{H} 4$ & Q14624 & 110 & 6.51 & 103,521 & 10 & -1000000 \\
\hline Apolipoprotein E & P02649 & 317 & 5.65 & 36,246 & 59 & -2.11918 \\
\hline Haptoglobin & P00738 & 97 & 6.13 & 45,861 & 14 & -1.51291 \\
\hline Complement $\mathrm{C} 1 \mathrm{q}$ & P02746 & 92 & 6.12 & 26,933 & 22 & -1000000 \\
\hline Inter-alpha-trypsin inhibitor heavy chain $\mathrm{H} 4$ & Q14624 & 306 & 6.51 & 103,521 & 27 & 2.86546 \\
\hline Complement C4-A & P0C0L4 & 221 & 6.65 & 194,247 & 12 & 2.26642 \\
\hline Complement $\mathrm{C} 3$ & P01024 & 179 & 6.02 & 188,569 & 9 & 4.58277 \\
\hline Serum albumin & P02768 & 136 & 5.92 & 71,317 & 25 & 3.20345 \\
\hline Serotransferrin & P02787 & 113 & 6.81 & 79,280 & 16 & 1000000 \\
\hline Haptoglobin & P00738 & 195 & 6.13 & 45,861 & 26 & 1000000 \\
\hline Keratin, type II cytoskeletal & P04264 & 549 & 6.52 & 76,356 & 17 & 1000000 \\
\hline Antithrombin-III & P01008 & 99 & 5.98 & 53,025 & 19 & 1000000 \\
\hline
\end{tabular}

Mw, molecular weight; PI, Isoelectric point; SV, stable vitiligo; C, control. The negative sign of SV/C ration means downregulated protein expression. The positive sign of SV/C ration means upregulated protein expression. A ratio of 1000000 means that the protein only appeared in the stable stage of vitiligo, but not appeared in the healthy volunteers and a ratio of -1000000 means that the protein only appeared in the healthy volunteers, but not in the stable stages of vitiligo.

Table III. Differentially expressed proteins occurring in both SV and PV.

\begin{tabular}{|c|c|c|c|c|c|c|}
\hline Protein name & Accession & Score & PI & $\mathrm{Mw}, \mathrm{kDa}$ & Percent coverage, $\%$ & PV/SV (Ratio) \\
\hline Mannose-binding protein $\mathrm{C}$ & P11226 & 65 & 5.39 & 26,526 & 26 & -1000000 \\
\hline Haptoglobin & P00738 & 173 & 6.13 & 45,861 & 21 & -2.76835 \\
\hline Complement C4-A & P0C0L4 & 158 & 6.65 & 194,247 & 8 & 1.85913 \\
\hline Ig mu chain $\mathrm{C}$ region & P01871 & 205 & 6.35 & 49,960 & 16 & 1.70843 \\
\hline Complement C4-B & P0C0L5 & 244 & 6.73 & 194,212 & 11 & 1.56144 \\
\hline Serum albumin & P02768 & 131 & 5.92 & 71,317 & 22 & 1000000 \\
\hline Immunoglobulin $\mathrm{J}$ chain & P01591 & 144 & 5.12 & 18,543 & 32 & 1.64301 \\
\hline
\end{tabular}

Mw, molecular weight; PI, Isoelectric point; SV, stable vitiligo; PV, progressive vitiligo. The negative sign of SV/PV ration means down-regulated protein expression. The positive sign of SV/PV ration means up-regulated protein expression. A ratio of 1000000 means that the protein only appeared in the progressive stage of vitiligo, but not in the stable stage of vitiligo and a ratio of -1000000 means that the protein only appeared in the stable stage of vitiligo, but not in the progressive stage of vitiligo.

Functional categories of identified proteins. An analysis of the functional categories of the proteins differentially expressed in patients with vitiligo compared to the controls was performed. The differentially expressed proteins in the SV and PV groups were analyzed based on their GO clustering. The annotated functions based on the GO analysis were ranked according to statistical significance. The differentially expressed proteins were categorized based on their molecular function, biological process, and cell component.

In regard to the molecular function, we noted that normal enzyme activity, ion binding and lipid binding function most frequently occurred at both stages of vitiligo compared to controls. In addition, we noted oxidoreductase activity, transmembrane transporter activity and DNA binding function (Fig. 1).
We further analyzed the GO annotated biological processes in vitiligo patients compared to controls. The top three biological processes were response to stress, vesicle-mediated transport and immune system biological process. We also observed other processes annotated as homeostatic, signal transduction, cell adhesion and small molecule metabolic processes (Fig. 2).

The cellular component GO terms associated with vitiligo compared to controls included extracellular components, organelle, plasma membrane, cytoplasm and endosome components (Fig. 3).

Western blot results. Compared to the samples from the control group, the samples from patients with vitiligo showed differential expression patterns of Peroxiredoxin-6 (PRDX6), 


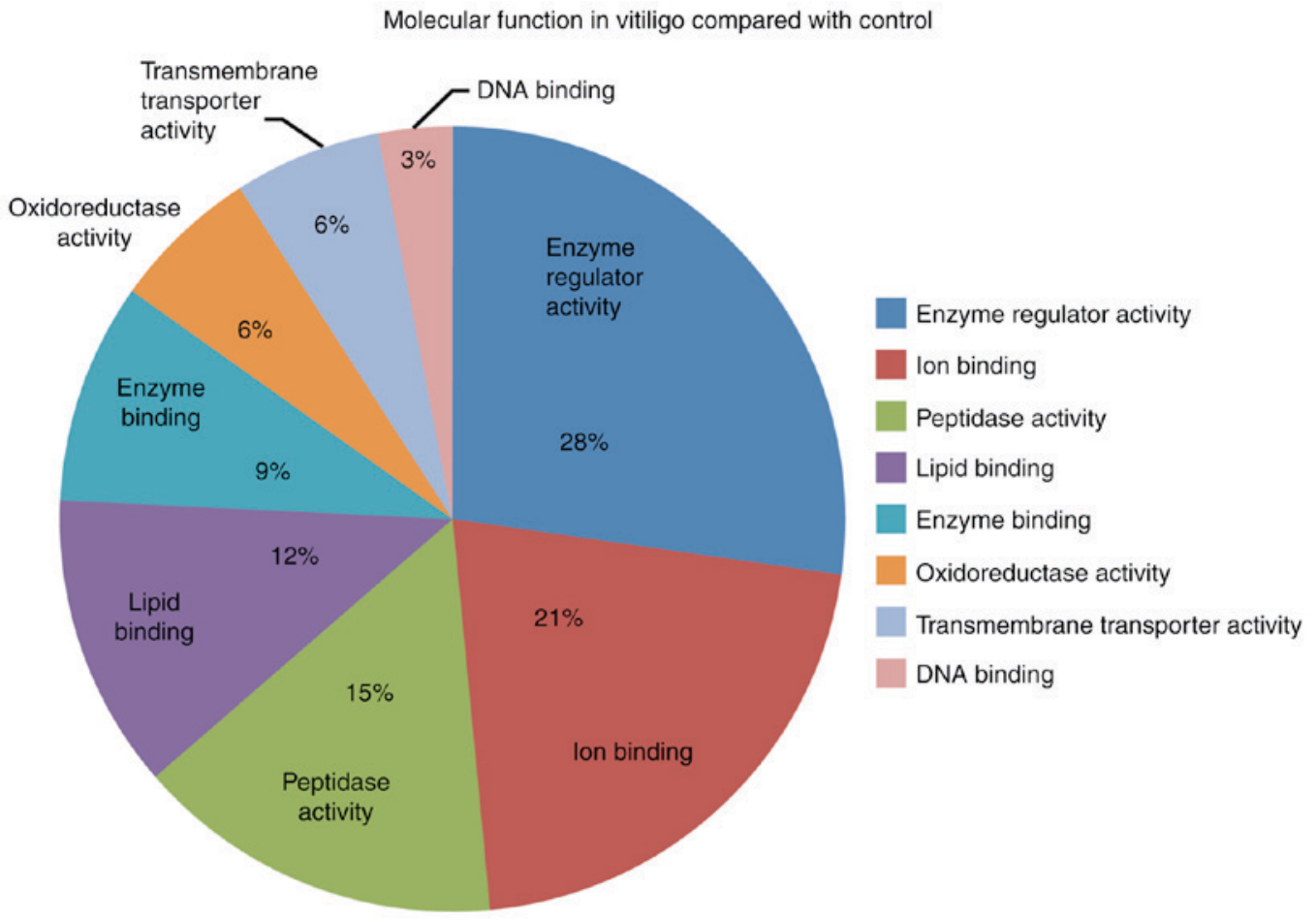

Figure 1. Molecular functions of the identified proteins in vitiligo compared with controls (ion binding, enzyme regulator and DNA binding occupy most of the proportion).

Biological process in vitiligo compared with control

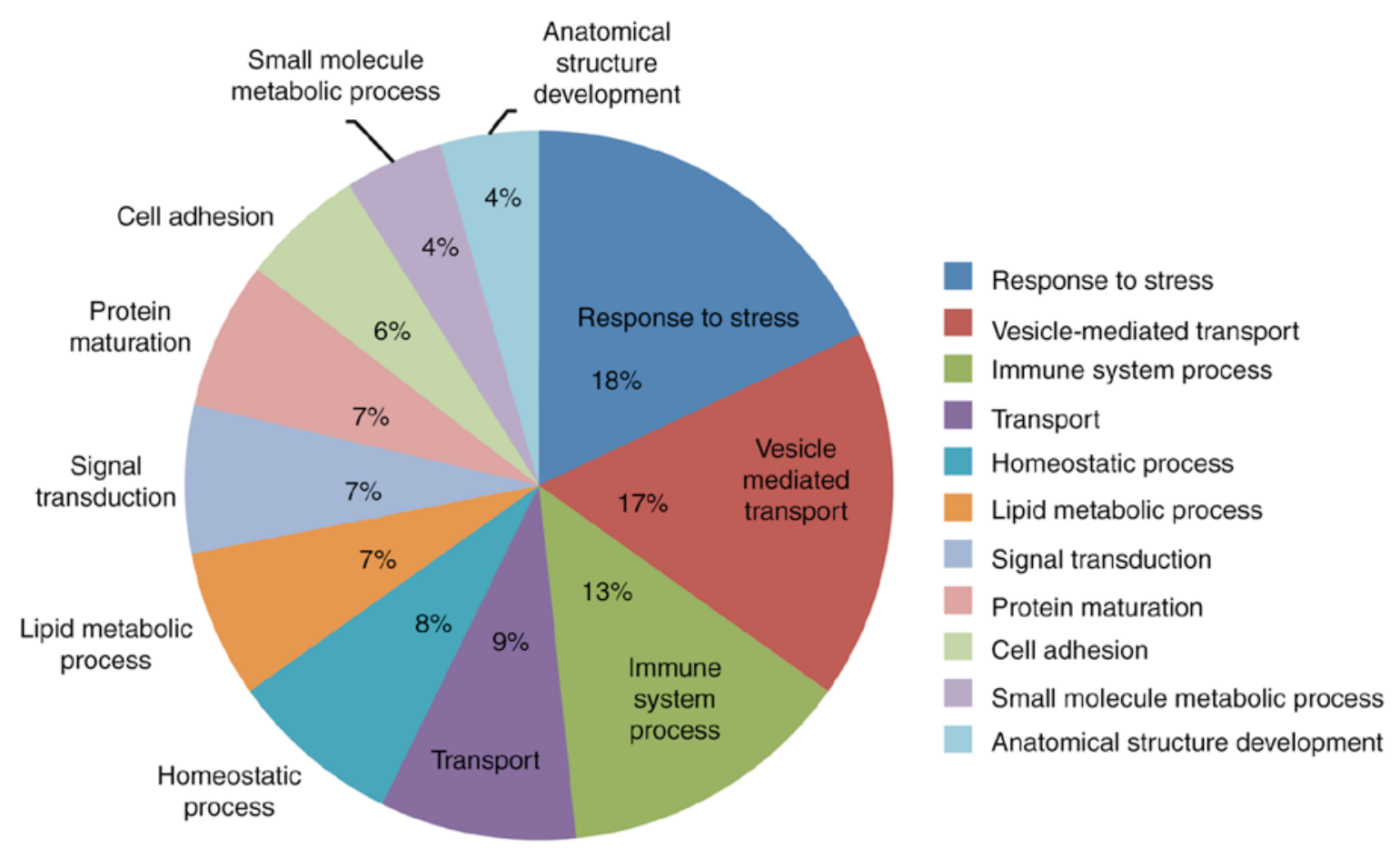

Figure 2. Biological process of identified proteins in vitiligo compared with controls (response to stress, vesicle-mediated transport and immune system process occupy most of the proportion).

apolipoprotein L1 (APOL1), apolipoprotein E (APOE) and mannose-binding protein C (MBL2). PRDX6 expression was downregulated in patients with vitiligo compared to that in healthy individuals (progressive vs. control was $\mathrm{P}<0.01$ ). In addition, APOL1 expression was downregulated in patients with PV compared to that in controls $(\mathrm{P}<0.05)$, and APOE expression was downregulated in patients with SV compared to that in controls $(\mathrm{P}<0.05)$. MBL2 was downregulated in 
Cellular component in vitiligo compared with control

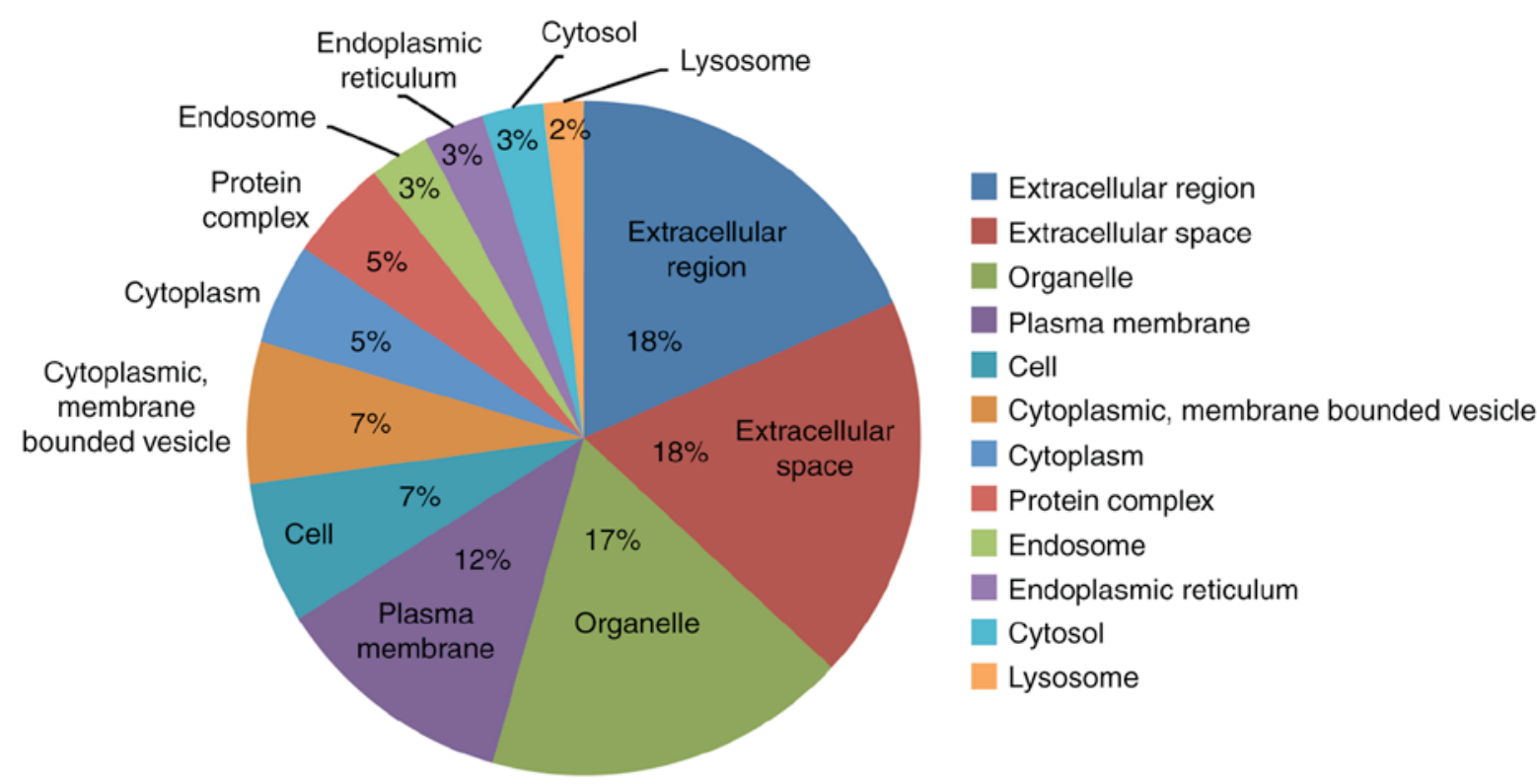

Figure 3. Cellular components of the identified proteins in vitiligo compared with controls (extracellular region, extracellular space and organelle occupy most of the proportion).

patients with PV compared to that in controls, especially compared to that in patients with SV (P<0.001; Fig. 4).

\section{Discussion}

The onset of vitiligo is related to oxidative stress, autoimmune responses, and neurogenic factors (11). Some studies have shown that sympathetic nervous system dysfunction can inhibit melanin production, leading to melanin loss (12). The incidence of nonsegmental vitiligo and autoimmune responses is closely related, and inflammatory cell infiltration can be found around the lesions of patients with segmental vitiligo (13). In addition, patients with vitiligo show increased hydrogen peroxide levels, and the resulting oxidative stress damage can cause melanocytes to become more susceptible to free radical-mediated damage (14). However, the pathogenesis of vitiligo is still largely unclear.

In this study, we used 2-DE-MS to identify proteins that are differentially expressed between patients with vitiligo and healthy individuals. The study identified many differentially expressed proteins, and it also showed a protein expression difference among patients with the progressive and stable stages of the disease. Analysis of the functions of the differentially expressed proteins using Cytoscape software showed that these proteins were involved in GO annotated molecular functions, biological processes, and cellular components. We found common functions that were to be expected, such as immune system processes (15) and oxidoreductase activities (16), but we also found new functions such as calcium-binding proteins, lipid binding proteins, peptidase enzyme activities and cell adhesion processes. There have been reports about serum vitamin D levels relating to skin diseases including vitiligo (17), and some authors have suggested that total lipid serum levels change in patients with vitiligo (18). However, no one has elucidated the mechanism of vitiligo or implicated specific proteins in its pathogenesis. Our data also suggest that new functions and biological processes involving these different proteins may be affected during the two stages of vitiligo. In addition, we found most of these proteins were expected to be localized to the extracellular region, the organelles, the plasma membrane and the cytoplasm.

We noted four proteins that were differentially expressed between vitiligo patients and controls: PRDX6, APOL1, APOE and mannose-binding protein C (MBL2). PRDX6 was significantly downregulated in patients with vitiligo, APOL1 was downregulated in patients with PV, and APOE was downregulated in patients with SV. Additionally, mannose-binding protein C (MBL2) was downregulated in patients with PV compared to that in patients with SV. We verified that PRDX6 was downregulated in both stages of vitiligo, especially in PV. Using our bioinformatic analysis, we showed evidence that PRDX6 might have oxidoreductase activity based on the biological function according to GO annotation. PRDX6 represents a widely distributed group of peroxiredoxins that contain a single conserved cysteine in the protein monomer (19), which protects tissues from oxidative stress. There have been reports that PRDX6 plays a role in protecting epithelial cells from exposure to severe oxidative stress (20). Another study on PRDX6 examined its expression levels in retinal pigment epithelial cells and showed that PRDX6 protects cells from $\mathrm{H}_{2} \mathrm{O}_{2}$-induced oxidative stress and apoptosis through the PI3K/AKT pathway (21). Thus, PRDX6 may impact antioxygenation. In addition, there have been reports that downregulation of PRDX6 was verified in DNA vaccine-induced mouse models of vitiligo by western-blot and mRNA analysis (22). We also have found that PRDX6 expression varied in vitiligo patients compared to that in controls using 2-DE proteomics. The downregulation of PRDX6 was also observed. Further study will be needed to confirm whether PRDX6 levels are affected in the vitiligo disease state, especially during the progressive stage. 


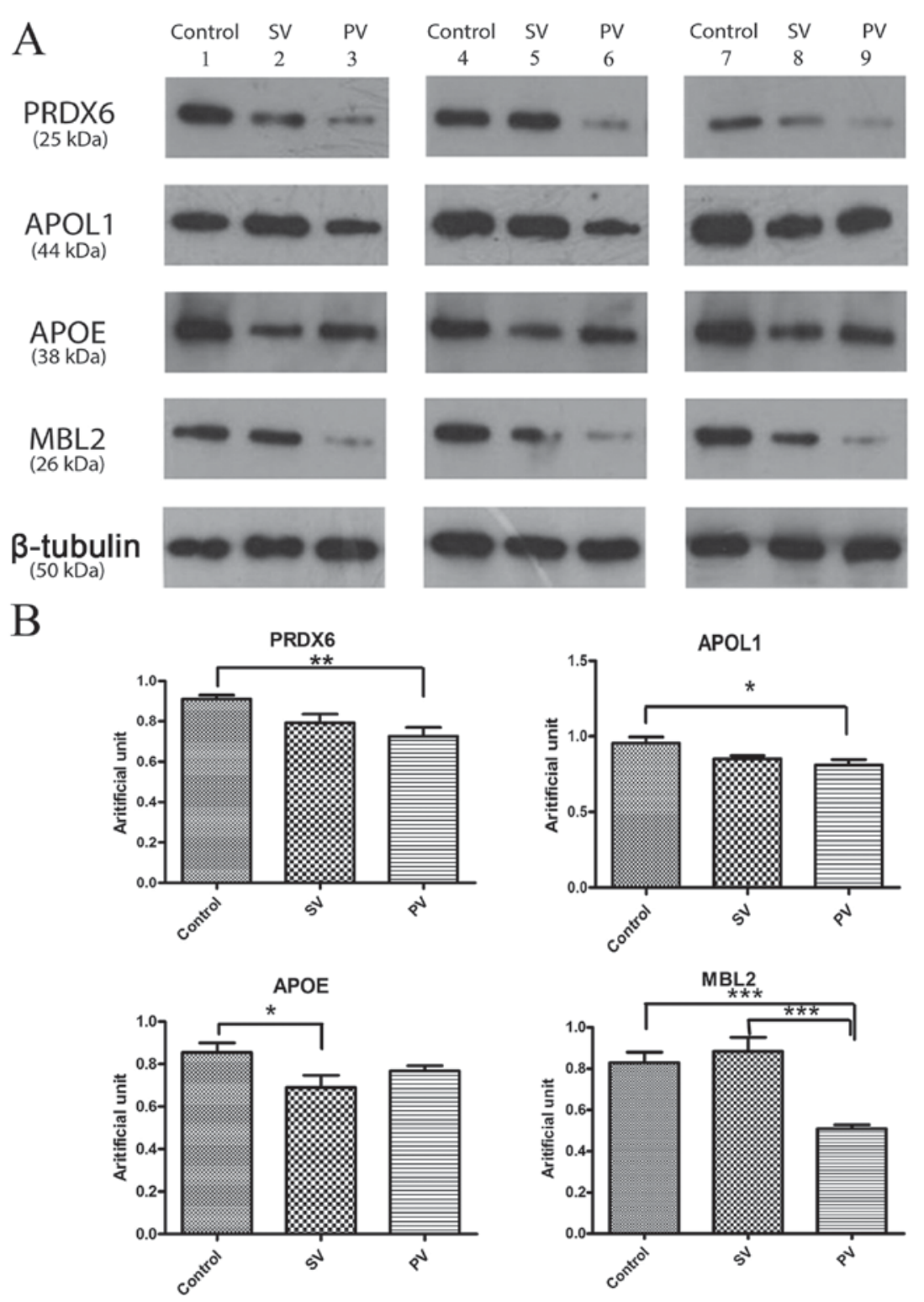

Figure 4. Four differential proteins expression of PRDX6, apolipoprotein (APOL1 and APOE) and mannose-binding protein C (MBL2). (A) Each column represents one group, and this group contains one control, one stable stage patient and one progressive stage patient sample. and beta-Tubulin was the loading control. They were the same loading quantity of protein sample. (B) The gray value ratio of each band compared between groups (stable stage or progressive stage compared with control) was used to perform the significance of differences by Student's t-test. P-value $<0.05$ was considered significant.

We also found variation in the expression of lipid metabolism-related proteins. APOA1 and APOL1 were significantly downregulated in patients with PV, and APOE was downregulated in patients with SV. APOA1 is the main apolipoprotein of high-density lipoprotein (HDL), which can help reverse cholesterol transport and has antioxidative and anti-inflammatory effects (23). The level of APOA1 is inversely correlated with the level of acute inflammation markers, (24) and studies have suggested that APOA1 can antagonize lipid deposition and anti-inflammatory-induced lipid toxicity (25). The APOL1 family mainly binds to APOA1 complex lipoproteins. Additionally, APOE, which was decreased during the stable stage, is involved in the transport of cholesterol, regulating lipid metabolism, and some neurobiological processes (26). APOE is an arginine-rich alkaline protein that is the APO component of various lipoproteins and acts as an important cholesterol carrier mainly in chylomicrons with very-low-density lipoprotein and certain HDLs $(27,28)$. In recent years, APOE has been reported to be a marker of ovarian cancer and to promote tumor growth (29). In addition, APOE is also involved in nerve repair and regeneration after injury. Our western blot results showed that APOL1 and APOE had significant differences in progressive and SV. Although it had not been reported that these proteins are related to lipid function in the context of vitiligo, there may some effect on lipid function in the vitiligo disease state. This information further illustrates that APOL1 and APOE may have an effect in the two stages of vitiligo by regulating LDL and HDL.

Notably, our results showed that MBL2 is downregulated in patients with PV compared with control and SV, suggesting that complement activation was inhibited. MBL2 is an acute-phase protein in the $\mathrm{Ca}^{2+}$-dependent lectin family produced by the liver. MBL is a key factor in the innate immune system that recognizes pathogenic microorganisms through 
the carbohydrate recognition domain (30,31). Furthermore, by combining with associated serine protease- 2 , the complement lectin pathway can be activated and result in immune responses and regulation of inflammation to mediate infections, immune diseases, and recurrent miscarriage diseases (32). MBL has been shown to affect immune diseases; for example, MBL is decreased in hypothyroidism during pregnancy (33) and SLE via polymorphisms (34). It was reported that a polymorphism in MBL2 may be one of the genetic susceptibility factors for vitiligo, but this is still largely an open question. Our bioinformatic classification of these proteins may help unravel how the ion-binding function of MBL2 plays a part in vitiligo (35).

We have found some differential expressed proteins in vitiligo compared to control using proteomics. The expression changes of these proteins through the two stages of vitiligo may yield some insight into how these proteins play important roles in the occurrence and development of vitiligo. In addition, the differential protein expression between the two stages may be an indicator of disease activity, and this information may one day provide potential clues in the clinical treatment of vitiligo. Subsequent further research is required to determine the clinical significance of the findings.

In summary, although our sample size was small, our results confirmed the differential expression of PRDX6, APOL1, and APOE. This information might be useful to study pathogenesis or biomarkers of stable and PV. MBL2 may have applications as a biological marker used to differentiate between the progressive and stable stages of the disease. Of course, these are only preliminary data and further research is required to validate the findings and verify the identified novel molecular biomarkers in patients with vitiligo. Based on the findings of this study, we will use other biological techniques to conduct a more in-depth exploration of protein interactions, protein modifications, and biological information networks to further elucidate the pathogenic mechanisms of vitiligo.

\section{Acknowledgements}

This study was supported by the Public Welfare Programme, Ministry of Health, China (no. 201202013), and the Innovative research team in universities, Liaoning Bureau of Education (no. LT2012012).

\section{References}

1. Isenstein AL, Morrell DS and Burkhart CN: Vitiligo: Treatment approach in children. Pediatr Ann 38: 339-344, 2009.

2. Guerra L, Dellambra E, Brescia S and Raskovic D: Vitiligo: Pathogenetic hypotheses and targets for current therapies. Curr Drug Metab 11: 451-467, 2010.

3. Birlea SA, Fain PR and Spritz RA: A Romanian population isolate with high frequency of vitiligo and associated autoimmune diseases. Arch Dermatol 144: 310-316, 2008.

4. Shin J, Kang HY, Kim KH, Park CJ, Oh SH, Lee SC, Lee S, Choi GS and Hann SK: Involvement of T cells in early evolving segmental vitiligo. Clin Exp Dermatol 41: 671-674, 2016.

5. Wang XX, Wang QQ, Wu JQ, Jiang M, Chen L, Zhang CF and Xiang LH: Increased expression of CXCR3 and its ligands in patients with vitiligo and CXCL10 as a potential clinical marker for vitiligo. Br J Dermatol 174: 1318-1326, 2016.

6. Richmond JM, Bangari DS, Essien KI, Currimbhoy SD, Groom JR, Pandya AG, Youd ME, Luster AD and Harris JE: Keratinocyte-derived chemokines orchestrate T-Cell positioning in the epidermis during vitiligo and may serve as biomarkers of disease. J Invest Dermatol 137: 350-358, 2017.
7. Speeckaert R, Lambert J, Grine L, Van Gele M, De Schepper S and van Geel N: The many faces of interleukin-17 in inflammatory skin diseases. Br J Dermatol 175: 892-901, 2016.

8. Guan M, Chen X, Ma Y, Tang L, Guan L, Ren X, Yu B, Zhang W and Su B: MDA-9 and GRP78 as potential diagnostic biomarkers for early detection of melanoma metastasis. Tumour Biol 36: 2973-2982, 2015.

9. Kazemipour N, Qazizadeh H, Sepehrimanesh M and Salimi S: Biomarkers identified from serum proteomic analysis for the differential diagnosis of systemic lupus erythematosus. Lupus 24: 582-587, 2015.

10. Group CAolMoDPCPDS: Consensus of Vitiligo Diagnosis and Treatment (2014 Edition). Chin J Dermatol 47: 65, 2014.

11. Jin Y, Birlea SA, Fain PR, Ferrara TM, Ben S, Riccardi SL, Cole JB, Gowan K, Holland PJ, Bennett DC, et al: Genome-wide association analyses identify 13 new susceptibility loci for generalized vitiligo. Nat Genet 44: 676-680, 2012.

12. Alikhan A, Felsten LM, Daly M and Petronic-Rosic V: Vitiligo: A comprehensive overview part I. Introduction, epidemiology, quality of life, diagnosis, differential diagnosis, associations, histopathology, etiology and work-up. J Am Acad Dermatol 65: 473-491, 2011.

13. Vaccaro M, Cannavò SP, Imbesi S, Cristani M, Barbuzza O, Tigano V and Gangemi S: Increased serum levels of interleukin-23 circulating in patients with non-segmental generalizedvitiligo. Int J Dermatol 54: 672-674, 2015.

14. Jian Z, Li K, Liu L, Zhang Y, Zhou Z, Li C and Gao T: Heme oxygenase-1 protects human melanocytes from $\mathrm{H} 2 \mathrm{O} 2$-induced oxidative stress via the Nrf2-ARE pathway. J Invest Dermatol 131: 1420-1427, 2011.

15. Rork JF, Rashighi M and Harris JE: Understanding autoimmunity of vitiligo and alopecia areata. Curr Opin Pediatr 28: 463-469, 2016.

16. Laddha NC, Dwivedi M, Mansuri MS, Singh M, Gani AR, Yeola AP, Panchal VN, Khan F, Dave DJ, Patel A, et al: Role of oxidative stress and autoimmunity in onset and progression of vitiligo. Exp Dermatol 23: 352-353, 2014.

17. Wadhwa B, Relhan V, Goel K, Kochhar AM and Garg VK: Vitamin D and skin diseases: A review. Indian J Dermatol Venereol Leprol 81: 344-355, 2015.

18. Dammak I, Boudaya S, Ben Abdallah F, Turki H, Attia H and Hentati B: Antioxidant enzymes and lipid peroxidation at the tissue level in patients with stable and active vitiligo. Int J Dermatol 48: 476-480, 2009.

19. Fisher AB: Peroxiredoxin 6 in the repair of peroxidized cell membranes and cell signaling. Arch Biochem Biophys 617: 68-83, 2017.

20. Shibata S, Shibata N, Shibata T, Sasaki H, Singh DP and Kubo E: The role of Prdx6 in the protection of cells of the crystalline lens from oxidative stress induced by UV exposure. Jpn J Ophthalmol 60: 408-418, 2016.

21. Zha X, Wu G, Zhao X, Zhou L, Zhang H, Li J, Ma L and Zhang Y: PRDX6 protects ARPE-19 cells from oxidative damage via PI3K/AKT signaling. Cell Physiol Biochem 36: 2217-2228, 2015 .

22. Zhou Q, Wang F, Zhang Y, Yang F, Wang Y and Sun S: Down-regulation of Prdx6 contributes to DNA vaccine induced vitiligo in mice. Mol Biosyst 7: 809-816, 2011.

23. Filou S, Lhomme M, Karavia EA, Kalogeropoulou C, Theodoropoulos V, Zvintzou E, Sakellaropoulos GC, Petropoulou PI, Constantinou C, Kontush A and Kypreos KE: Distinct roles of apolipoproteins $\mathrm{A} 1$ and $\mathrm{E}$ in the modulation of high-density lipoproteincomposition and function. Biochemistry 55: 3752-3762, 2016.

24. Fizelova M, Miilunpohja M, Kangas AJ, Soininen P, Kuusisto J, Ala-Korpela M, Laakso M and Stančáková A: Associations of multipie lipoprotein and apolipoprotein measures with worsening of glycemia and incident type 2 diabetes in 6607 non-diabetic Finnish men. Atherosclerosis 240: 272-277, 2015.

25. Simó R, García-Ramírez M, Higuera M and Hernández C: Apolipoprotein A1 is overexpressed in the retina of diabetic patients. Am J Ophthalmol 147: 319-325.e1, 2009.

26. Meroufel DN, Mediene-Benchekor S, Lardjam-Hetraf SA, Ouhaïbi-Djellouli H, Boulenouar H, Hamani-Medjaoui I, Hermant X, Saïdi-Mehtar N, Amouyel P, Houti L, et al: Associations of common SNPs in the SORT1, GCKR, LPL, APOA1, CETP, LDLR, APOE genes with lipid trait levels in an Algerian population sample. Int J Clin Exp Pathol 8: 7358-7363, 2015. 
27. Ikeda T, Shinohata R, Murakami M, Hina K, Kamikawa S, Hirohata S, Kusachi S, Tamura A and Usui S: A rapid and precise method for measuring plasma apoE-rich HDL using polyethylene glycol and cation-exchange chromatography: A pilot study on the clinical significance of apoE-rich HDL measurements. Clin Chim Acta 465: 112-118, 2017.

28. Dai S, Wang B, Li W, Wang L, Song X, Guo C, Li Y, Liu F, Zhu F, Wang Q, et al: Systemic application of 3-methyladenine markedly inhibited atherosclerotic lesion in ApoE-/-mice by modulating autophagy, foam cell formation and immune-negative molecules. Cell Death Dis 7: e2498, 2016.

29. Urquidi V, Goodison S, Ross S, Chang M, Dai Y and Rosser CJ: Diagnostic potential of urinary $\alpha 1$-antitrypsin and apolipoprotein E in the detection of bladder cancer. J Urol 188: 2377-2383, 2012.

30. Orsatti CL, Nahas EA, Nahás-Neto J, Orsatti FL, Linhares IM and Witkin SS: Mannose-binding lectin gene polymorphism and risk factors for cardiovascular disease in postmenopausal women. Mol Immunol 61: 23-27, 2014.

31. Heitzeneder S, Seidel M, Förster-Waldl E and Heitger A: Mannan-binding lectin deficiency-Good news, bad news, doesn't matter? Clin Immunol 143: 22-38, 2012.
32. Swierzko AS, Szala A, Sawicki S, Szemraj J, Sniadecki M, Sokolowska A, Kaluzynski A, Wydra D and Cedzynski M: Mannose-binding lectin (MBL) and MBL-associated serine protease-2 (MASP-2) in women with malignant and benign ovarian tumours. Cancer Immunol Immunother 63: 1129-1140, 2014.

33. Çalkavur, Ş Erdemir G, Onay H, AltunKöroğlu Ö, Yalaz M, Žekioğlu O, Aksu G, Özkınay F, Akercan F and Kültürsay N: Mannose-binding lectin may affect pregnancy outcome. Turk J Pediatr 57: 26-33, 2015.

34. Pradhan V, Surve P, Rajadhyaksha A, Rajendran V,Patwardhan M, Umare V, Ghosh K and Nadkarni A: Mannose binding lectin (MBL) 2 gene polymorphism \& its association with clinical manifestations in systemic lupus erythematosus (SLE) patients from western India. Indian J Med Res 141: 199-204, 2015.

35. Onay H, Pehlivan M, Alper S, Ozkinay F and Pehlivan S: Might there be a link between mannose binding lectin and vitiligo? Eur J Dermatol 17: 146-148, 2007. 